TRANSACTIONS OF THE

AMERICAN MATHEMATICAL SOCIETY

Volume 353, Number 3, Pages 1039-1054

S 0002-9947(00)02647-7

Article electronically published on August 8, 2000

\title{
TRANSFERS OF CHERN CLASSES IN BP-COHOMOLOGY AND CHOW RINGS
}

\author{
BJÖRN SCHUSTER AND NOBUAKI YAGITA
}

\begin{abstract}
The $B P^{*}$-module structure of $B P^{*}(B G)$ for extraspecial 2-groups is studied using transfer and Chern classes. These give rise to $p$-torsion elements in the kernel of the cycle map from the Chow ring to ordinary cohomology first obtained by Totaro.
\end{abstract}

\section{INTRODUCTION}

Let $G$ be a compact Lie group, e.g. a finite group, and $B G$ its classifying space. For complex oriented cohomology theories $h$ one can define in $h^{*}(B G)$ Chern classes of complex representations of $G$, and also transfer maps. We are interested in the Mackey closure $\overline{C h}_{h}(G)$ of the ring of Chern classes in $h^{*}(B G)$, namely the subring of $h^{*}(B G)$ recursively generated by transfers of Chern classes. By [HKR], this is equal to the $h^{*}$-module generated by transfers of Euler classes.

For ordinary mod $p$ cohomology, Green and Leary [GL] showed that the inclusion map $i: \overline{C h}_{H \mathbb{Z} / p} \hookrightarrow H^{*}(B G ; \mathbb{Z} / p)$ is an F-isomorphism, i.e., the induced map of varieties is a homeomorphism. Green and Minh [GM], however, noticed that $i / \sqrt{0}$ need not be an isomorphism in general. Next consider $h=B P$ or $h=K(n)$, the $n$-th Morava $K$-theory, at a fixed prime $p$. Following Hopkins, Kuhn and Ravenel [HKR, we shall call a group $G$ "good" for $h$-theory if $h^{*}(B G)$ is generated (as an $h^{*}$ module) by transferred Euler classes of representations of subgroups of $G$. It is clear that if the Sylow $p$-subgroup of $G$ is good, then so is $G$, and one has an isomorphism $h^{*}(B G) \cong \overline{C h}_{h}(G)$. Furthermore, it follows from [RWY] that $G$ is good for $B P$ if it is good for $K(n)$ for all $n$. Examples for groups that are $K(n)$-good for all $n$ are the finite symmetric groups. Another typical case are $p$-groups of $p$-rank at most 2 and $p \geq 5$ : in [Y1] it is shown that the Thom map $\rho: B P^{*}(-) \rightarrow H^{*}(-)_{(p)}$ induces an isomorphism $B P^{*}(B G) \otimes_{B P^{*}} \mathbb{Z}_{(p)} \cong H^{\text {even }}(B G)$. Note however that I. Kriz claimed that $K(n)^{\text {odd }}(B G) \neq 0$ for some $p$-groups $G$.

On the other hand, B. Totaro T1 found a way to compare $B P$-theory to the Chow ring. For a complex algebraic variety $X$, the groups $C H^{i}(X)$ of codimension $i$ algebraic cycles modulo rational equivalence assemble to the Chow ring $C H^{*}(X)=$ $\sum_{i} C H^{i}(X)$. Totaro constructed a map $\tilde{\rho}: C H^{*}(X) \rightarrow B P^{*}(X) \otimes_{B P^{*}} \mathbb{Z}_{(p)}$ such that the composition

$$
\bar{\rho}: C H^{i}(X)_{(p)} \stackrel{\tilde{\rho}}{\longrightarrow} B P^{*}(X) \otimes_{B P^{*}} \mathbb{Z}_{(p)} \stackrel{\rho}{\longrightarrow} H^{*}(X)_{(p)}
$$

Received by the editors March 29, 1999.

2000 Mathematics Subject Classification. Primary 55P35, 57T25; Secondary 55R35, 57T05.

Key words and phrases. BP-theory, transfer, Chern classes, Chow ring. 
coincides with the cycle map. One of the main results of [T1] is that there exists a group $G$ for which the kernel of $\bar{\rho}$ contains $p$-torsion elements. To prove this, Totaro defined the Chow ring of a classifying space $B G$ as $\operatorname{Lim}_{m \rightarrow \infty} C H^{*}\left(\left(\mathbb{C}^{m}-S\right) / G\right)$, where $G$ acts on $\mathbb{C}^{m}-S$ freely and $\operatorname{codim}(S) \rightarrow \infty$ as $m \rightarrow \infty$. He then constructed a non-zero element $x$ in $\operatorname{Ker}(\rho)$ such that

$$
x \in \operatorname{Im}\left(\overline{C h}_{B P}(B G) \rightarrow\left(B P^{*}(B G) \otimes_{B P^{*}} \mathbb{Z}_{(p)}\right)\right) .
$$

Since transfers and Chern classes also exist in the Chow ring $C H^{*}(B G)$, there is an element $\bar{x} \in \overline{C h}_{C H}(G)$ that also lies in $\operatorname{Ker}(\bar{\rho})$. The group Totaro uses is $G=\mathbb{Z} / 2 \times D_{+}^{1+4}$, where $D_{+}^{1+4}=D(2)$ is the extraspecial 2-group of order 32, which is isomorphic to the central product of two copies of the dihedral group $D_{8}$ of order 8. He first proves that there exists an element $x \in B P^{*}(B D(2))$ satisfying (1.1) but which restricts to zero under the map $\rho_{\mathbb{Z} / 2}: B P^{*}(-) \rightarrow H^{*}(-; \mathbb{Z} / 2)$, where he uses the computation of $B P^{*}(B S O(4))$ from $[\mathrm{KY}$.

Let $D(n)=2_{+}^{1+2 n}$ denote the extraspecial 2-group of order $2^{2 n+1}$; it is isomorphic to the central product of $n$ copies of $D_{8}$. In this paper, we construct non-zero elements $x \in B P^{*}(B D(n))$ satisfying (1.1) but with $\rho_{\mathbb{Z} / 2}(x)=0$ directly for each $n$.

Let $\tilde{W}$ be a maximal elementary abelian 2-subgroup and $N$ the center of $D(n)$. For a one-dimensional real representation $e$ of $\tilde{W}$ restricting non-trivially to the center, set $\Delta=\operatorname{Ind}_{\tilde{W}}^{D(n)}(e)$. This is the unique irreducible representation which acts non-trivially on $N$. Then the $i$-th Stiefel-Whitney class $w_{i}(\Delta)$ for $i<2^{n}$ can be written as a polynomial in variables $w_{1}\left(e_{j}\right), 1 \leq j \leq 2 n$, for 1-dimensional representations $e_{j}$ of $D(n) / N\left(\left[\mathbf{Q}\right.\right.$, Remark 5.13), i.e. $w_{i}(\Delta)=w_{i}\left(w_{1}\left(e_{1}\right), \ldots, w_{1}\left(e_{2 n}\right)\right)$. Let $e_{\mathbb{C}}^{\prime}$ denote the complex representation induced from the real representation $e^{\prime}$. Then we can prove that

$$
x=c_{2^{n-1}}\left(\Delta_{\mathbb{C}}\right)-w_{2^{n-1}}\left(c_{1}\left(e_{1 \mathbb{C}}\right), \ldots, c_{1}\left(e_{2 n \mathbb{C}}\right)\right)
$$

satisfies (1.1) together with $\rho_{\mathbb{Z} / 2}(x)=0$, and furthermore conclude that $\operatorname{Ker}(\rho) \neq 0$ for $G=\mathbb{Z} / 2 \times D(n)$.

Second, we construct a non-nilpotent element $x \in B P^{*}(B G)$ which is not in $\overline{C h}_{B P}(B G)$ and such that

$$
x \in \operatorname{Ker}(\rho) \text { and } 0 \neq x \in\left(B P^{*}(B G) \otimes_{B P^{*}} \mathbb{Z}_{(p)}\right) .
$$

However we do not know whether $x$ comes from the Chow ring or not, and we only obtain the result for $n=3,4$. Set

$$
x=\left[v_{1} \otimes w_{2^{n}}(\Delta)\right]
$$

to be the class represented by $v_{1} \otimes w_{2^{n}}(\Delta)$ in the $E_{\infty}$-page of the Atiyah-Hirzebruch spectral sequence. If this element exists, then restricting to the center of $D(n)$ we see that $x$ is not in $\overline{C h}_{B P}(B G)$. However, it seems difficult to prove that this cycle is permanent. For the case $n=3,4$, we use $B P$-theory of $B \operatorname{Spin}(7)$ and $B \operatorname{Spin}(9)$ computed in $[\mathrm{KY}$ to see that $x$ is a permanent cycle.

These arguments do not seem to work for other extraspecial 2-groups, nor for 2 -groups that have a cyclic maximal normal subgroup [S].

In Section 2, we recall the mod 2 cohomology of extraspecial 2-groups following Q]. In particular, $w_{2^{n}-2^{i}}(\Delta)$ is represented by the Dickson invariant $D_{i}$, and we study the action of the Milnor primitives $Q_{j}$ on $D_{i}$. To see that $\rho(x) \neq 0$ in $H^{*}(B D(n) ; \mathbb{Z})$, we recall the integral cohomology in Section 3. In Section 4, we 
show that $x$ satisfies (1.1). In Section 5, we study how elements in $\operatorname{Ker}(\rho)$ are represented in the Atiyah-Hirzebruch spectral sequence, assuming some technical conditions which are satisfied in the cases $n=3$ and $n=4$. The element $x$ in (1.4) is proved not to be in $\overline{C h}_{B P}(B D(n))$ in Section 6. In Section 7 the element $x$ in (1.4) is proved to be a permanent cycle in the Atiyah-Hirzebruch spectral sequence for $n=3,4$ by comparing the spectral sequence to the corresponding spectral sequence for $H^{*}(B \operatorname{Spin}(2 n+1))$. The last section gives more examples of $p$-torsion elements in the kernel of the cycle map, using spinor groups and the exceptional group $F_{4}$.

We would like to thank Burt Totaro for pointing out some errors in an earlier version of this paper.

\section{Extraspecial 2-Groups}

The extraspecial 2-group $D(n)=2_{+}^{1+2 n}$ is the central product of $n$ copies of the dihedral group $D_{8}$ of order 8 . So there is a central extension

$$
0 \rightarrow N \longrightarrow D(n) \stackrel{\pi}{\longrightarrow} V \rightarrow 0
$$

with $N \cong \mathbb{Z} / 2$ and $V$ elementary abelian of rank $2 n$. Take a set of generators $c, \tilde{a}_{1}, \ldots, \tilde{a}_{2 n}$ of $D(n)$ such that $c$ is a generator of $N$, the elements $a_{i}=\pi\left(\tilde{a}_{i}\right)$ form a $\mathbb{Z} / 2$-basis of $V$, and

$$
\left[\tilde{a}_{j}, \tilde{a}_{2 i}\right]= \begin{cases}c & \text { if } j=2 i-1, \\ 0 & \text { else. }\end{cases}
$$

Using the Hochschild-Serre spectral sequence associated to extension (2.1), Quillen Q determined the mod 2 cohomology of $D(n)$. Let $e_{i}$ denote the real 1-dimensional representation of $D(n)$ given as the projection onto $\left\langle a_{i}\right\rangle$ followed by the nontrivial character $\left\langle a_{i}\right\rangle \rightarrow\{ \pm 1\} \subset \mathbb{R}$, and $e: \tilde{V}^{\text {odd }} \rightarrow N \rightarrow\{ \pm 1\} \subset \mathbb{R}$, where $\tilde{V}^{\text {odd }}=$ $\left\langle c, \tilde{a}_{2 i-1} \mid 1 \leq i \leq n\right\rangle$ is a maximal elementary abelian 2-subgroup of $D(n)$. Define classes $x_{i} \in H^{1}(D(n) ; \mathbb{Z} / 2), w_{2^{n}} \in H^{2^{n}}(D(n) ; \mathbb{Z} / 2)$ as the Euler classes of the $e_{i}$ and of $\Delta=\operatorname{Ind}_{\tilde{V} \text { odd }}^{D(n)}(e)$, respectively. The extension (2.1) is represented by the class $f=x_{1} x_{2}+\cdots+x_{2 n-1} x_{2 n}$, and one has

$$
H^{*}(B D(n) ; \mathbb{Z} 2) \cong \mathbb{Z} / 2\left[w_{2^{n}}\right] \otimes \mathbb{Z} 2\left[x_{1}, \ldots, x_{2 n}\right] /\left(f, Q_{0} f, \ldots, Q_{n-2} f\right),
$$

where the $Q_{i}$ are Milnor's operations recursively defined by $Q_{0}=S q^{1}$ and $Q_{i}=$ $\left[S q^{2^{i}}, Q_{i-1}\right]$. The extension class $f$ defines a quadratic form $q: V \rightarrow \mathbb{Z} / 2$ on $V$. A subspace $W \subset V$ is said to be $q$-isotropic if $q(x)=0$ for all $x \in W$. The maximal (elementary) abelian subgroups of $D(n)$ are in one-to-one correspondence with the maximal isotropic subspaces of $V$. Indeed, if $W$ is maximal isotropic, then $\tilde{W}:=\pi^{-1}(W) \cong N \oplus W$ is maximal (elementary) abelian. Quillen also proved that the mod 2 cohomology of $D(n)$ is detected on maximal elementary abelian subgroups, i.e. the restrictions define an injective map

$$
H^{*}(B D(n) ; \mathbb{Z} / 2) \hookrightarrow \prod H^{*}(\tilde{W} ; \mathbb{Z} / 2),
$$

where the product ranges over conjugacy classes of maximal elementary abelian subgroups. Since the restriction of $\Delta$ to any such $\tilde{W}$ is the real regular representation (see [Q], Section 5), we have

$$
\operatorname{Res}_{\tilde{W}}\left(w_{2^{n}}\right)=\prod_{x \in H^{1}(W ; \mathbb{Z} / 2)}(z+x)
$$


where $z$ denotes the generator of $H^{*}(N ; \mathbb{Z} / 2)$ dual to $c$. For simplicity, write $w^{\prime}=\operatorname{Res}_{\tilde{W}} w_{2^{n}}$, and choose generators of $H^{*}(W ; \mathbb{Z} / 2) \cong \mathbb{Z} / 2\left[x_{1}^{\prime}, \ldots, x_{n}^{\prime}\right]$. It is well-known that the right hand side of (2.4) can be written in terms of Dickson invariants,

$$
w^{\prime}=z^{2^{n}}+D_{1} z^{2^{n-1}}+\cdots+D_{n} z
$$

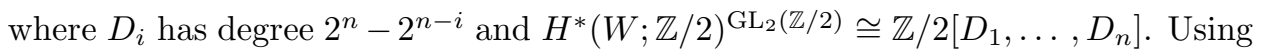
that the product of all the $x_{i}^{\prime}$ 's is clearly invariant and that the Milnor primitives are derivations, it is easy to see that the Dickson invariants may be written in terms of the $Q_{i}$ as follows:

$$
\begin{aligned}
D_{n} & =Q_{0} Q_{1} \ldots Q_{n-2}\left(x_{1}^{\prime} \cdots x_{n}^{\prime}\right) \\
D_{i} & =\left(Q_{0} \ldots \hat{Q}_{n-i-1} \ldots Q_{n-1}\left(x_{1}^{\prime} \cdots x_{n}^{\prime}\right)\right) / D_{n} .
\end{aligned}
$$

Lemma 2.1. The Milnor operations $Q_{1}, \ldots, Q_{n-1}$ act by

(1) $Q_{n-1} D_{i}=D_{n} D_{i}$;

(2) $Q_{n-j-1} D_{j}=D_{n}$;

(3) $Q_{i} D_{j}=0$ for $i<n-1$ and $i \neq n-j-1$.

Proof. First note that from (2.6) and $Q_{k}^{2}=0$ we immediately get $Q_{k}\left(D_{n}\right)=0$ for $k \neq n-1$, and $Q_{n-1} D_{n}=Q_{0} \ldots Q_{n-1}\left(x_{1}^{\prime} \cdots x_{n}^{\prime}\right)=D_{n}^{2}$. Thus, for each $1 \leq i \leq$ $n-1$,

$$
\begin{aligned}
0 & =Q_{n-1}\left(Q_{0} \ldots \hat{Q}_{n-i-1} \ldots Q_{n-1}\right)\left(x_{1}^{\prime} \cdots x_{n}^{\prime}\right)=Q_{n-1}\left(D_{i} D_{n}\right) \\
& =\left(Q_{n-1} D_{i}\right) D_{n}+D_{i} Q_{n-1} D_{n}=\left(Q_{n-1} D_{i}\right) D_{n}+D_{i} D_{n}^{2},
\end{aligned}
$$

whence (1). Similarly, (2) is implied by

$$
\begin{aligned}
D_{n}^{2} & =Q_{n-1} \cdots Q_{0}\left(x_{1}^{\prime} \cdots x_{n}^{\prime}\right)=Q_{n-i-1}\left(D_{i} D_{n}\right) \\
& =\left(Q_{n-i-1} D_{i}\right) D_{n}+D_{i} Q_{n-i-1} D_{n}=\left(Q_{n-i-1} D_{i}\right) D_{n} .
\end{aligned}
$$

Finally, for $k \neq n-i-1$ we get $0=Q_{k}\left(D_{i} D_{n}\right)=\left(Q_{k} D_{i}\right) D_{n}+D_{i} Q_{k} D_{n}=$ $\left(Q_{k} D_{i}\right) D_{n}$.

Corollary 2.2. $Q_{n-1} w^{\prime}=D_{n} w^{\prime}$ and $Q_{k} w^{\prime}=0$ for $k<n-1$.

Proof. For $j \neq n-1$, we have $Q_{j} w^{\prime}=\sum_{i=1}^{n-1}\left(Q_{j} D_{i}\right) z^{2^{n-i}}+Q_{j}\left(D_{n} z\right)=D_{n} z^{2^{j+1}}+$ $D_{n} z^{2^{j+1}}=0$. For $j=n-1$, we get $Q_{n-1} w^{\prime}=0+D_{n} D_{1} z^{2^{n-1}}+\cdots+D_{n} D_{n-1} z^{2}+$ $Q_{n-1}\left(D_{n} z\right)$. The last term equals $D_{n}^{2} z+D_{n} z^{2^{n}}$; the claim follows.

Corollary 2.3. $Q_{k} w_{2^{n}}=0$ for $0 \leq k \leq n-2$, but $Q_{n-1} w_{2^{n}} \neq 0$.

For future reference we note:

Lemma 2.4. $Q_{n}\left(D_{n} w^{\prime}\right)=D_{n}^{2} w^{\prime 2}, Q_{n}\left(D_{n-1} D_{n}\right)=D_{n}^{4}, Q_{n}\left(D_{n-1} w^{\prime}\right)=D_{n}^{3} w^{\prime}+$ $D_{n} D_{n-1}{w^{\prime}}^{2}, Q_{n+1} Q_{n}\left(D_{n-1} w^{\prime}\right)=D_{n}^{4} w^{\prime 4}$.

Proof. From (2.5) we see that $Q_{n}\left(D_{n} w^{\prime}\right)=\left(Q_{n} D_{n}\right) z^{2^{n}}+\sum_{i=1}^{n-1} Q_{n}\left(D_{n} D_{i}\right) z^{2^{n-i}}+$ $D_{n}^{2} z^{2^{n+1}}$. The coefficients of $z^{2^{n+1}}$ and $z$ tell us that this is equal to $D_{n}^{2}{w^{\prime}}^{2}$. Comparing coefficients further shows that $Q_{n}\left(D_{n}\right)=D_{1}^{2} D_{n}^{2}$ and $Q_{n}\left(D_{n} D_{i}\right)=D_{n}^{2} D_{i+1}^{2}$; in particular, $Q_{n}\left(D_{n} D_{n-1}\right)=D_{n}^{4}$. Thus we have

$$
\begin{aligned}
Q_{n}\left(D_{n} D_{n-1} D_{n} w^{\prime}\right) & =Q_{n}\left(D_{n} D_{n-1}\right) D_{n} w^{\prime}+D_{n} D_{n-1} Q_{n}\left(D_{n} w^{\prime}\right) \\
& =D_{n}^{5} w^{\prime}+D_{n}^{3} D_{n-1} w^{\prime 2} .
\end{aligned}
$$


Hence we get $Q_{n}\left(D_{n-1} w^{\prime}\right)=D_{n}^{3} w^{\prime}+D_{n} D_{n-1}{w^{\prime}}^{2}$. Next consider

$$
Q_{n+1}\left(D_{n} w^{\prime}\right)=\left(Q_{n+1} D_{n}\right) z^{2^{n}}+\sum_{i=1}^{n-1} Q_{n+1}\left(D_{n} D_{i}\right) z^{2^{n-i}}+D_{n}^{2} z^{2^{n+2}} .
$$

From the coefficients of $z^{2^{n+2}}$ and $z^{2^{n+1}}$, we see that $Q_{n+1}\left(D_{n} w^{\prime}\right)=D_{n}^{2} w^{\prime 4}+$ $D_{n}^{2} D_{1}^{4}{w^{\prime}}^{2}$ and hence $Q_{n+1}\left(D_{n} D_{n-1}\right)=D_{n}^{4} D_{1}^{4}$. Therefore

$$
Q_{n+1}\left(D_{n} D_{n-1} D_{n} w^{\prime}\right)=D_{n}^{4} D_{1}^{4} D_{n} w^{\prime}+D_{n} D_{n-1}\left(D_{n}^{2}{w^{\prime}}^{4}+D_{n}^{2} D_{1}^{4}{w^{\prime}}^{2}\right) .
$$

Thus $Q_{n+1}\left(D_{n-1} w^{\prime}\right)=D_{n}^{3} w^{\prime} D_{1}^{4}+D_{n} D_{n-1} w^{\prime 4}+D_{n} D_{n-1} D_{1}^{4} w^{\prime 2}$. Hence

$$
\begin{aligned}
Q_{n+1} Q_{n}\left(D_{n-1} w^{\prime}\right) & =Q_{n} Q_{n+1}\left(D_{n-1} w^{\prime}\right) \\
& =D_{n}^{4}{w^{\prime 2}}^{\prime 2} D_{1}^{4}+D_{n}^{4} w^{\prime 4}+D_{n}^{4} D_{1}^{4} w^{\prime 2}=D_{n}^{4}{w^{\prime}}^{4} .
\end{aligned}
$$

\section{The INTEGRAL COHOMOLOGY}

The integral cohomology of $D(n)$ is studied by Harada and Kono ([HK]; also see BC] ) by means of the Bockstein spectral sequence

$$
E_{1}=H^{*}(B G ; \mathbb{Z} / 2) \Longrightarrow \mathbb{Z} / 2 \otimes H^{*}(B G) /(2 \text {-torsion }) .
$$

The $E_{2}$-page of this spectral sequence is the $Q_{0}$-homology of $H^{*}(B G ; \mathbb{Z} / 2)$, and $E_{\infty} \cong \mathbb{Z} / 2$ for a finite group $G$. For $0 \leq i \leq n-2$, let

$$
R(i)=H^{*}(B V ; \mathbb{Z} / 2) /\left(f, Q_{0} f, \ldots, Q_{i} f\right) .
$$

Using the long exact sequence associated to the short exact sequence

$$
0 \rightarrow R(i-1) \stackrel{Q_{i} f}{\longrightarrow} R(i-1) \longrightarrow R(i) \rightarrow 0,
$$

Harada and Kono computed the $E_{2}$-page for $D(n)$ as follows:

$$
H\left(H^{*}(B D(n) ; \mathbb{Z} / 2) ; Q_{0}\right) \cong \Lambda\left(a, b_{1}, \ldots, b_{n-1}\right) \otimes \mathbb{Z} / 2\left[w_{2^{n}}\right],
$$

where $|a|=3$ and $\left|b_{i}\right|=2^{i}$. Since $E_{\infty} \cong \mathbb{Z} / 2$, the first non-trivial differential must be $d a=b_{1}$, and there have to be subsequent differentials $d\left(a b_{i}\right)=b_{i+1}$. Thus there appear exactly $n$ non-zero differentials in this spectral sequence. On the other hand, using corestriction arguments it is easy to see that the exponent of $H^{*}(B D(n))$ is at most $n+1$. Based on these facts, Harada and Kono proved the following.

Theorem $3.1([\mathrm{HK}])$. Let $C(n)^{*}=H^{*}(B D(n)) / J_{V}$, where $J_{V}$ is the ideal generated by the image of $H^{*}(B V)$ in $H^{*}(B D(n))$. Then $C(n)^{*} \subset H^{*}(B D(n))$, and there is an additive isomorphism

$$
C(n)^{k}= \begin{cases}\mathbb{Z} / 2^{\nu_{2}(k)} & \text { if } \nu_{2}(k) \leq n-1, \\ \mathbb{Z} / 2^{n+1} & \text { if } \nu_{2}(k)=n,\end{cases}
$$

where $\nu_{2}(k)$ denotes the 2 -adic valuation of $k$.

Let $c_{k}(n)$ denote a $\mathbb{Z}_{(2)}$-module generator of $C(n)^{2^{k}}$. Then $c_{n}(n)$ reduces to $w_{2^{n}}$ modulo $H^{*}(B V ; \mathbb{Z} / 2)$. Consider the restriction map $i: C(n)^{*} \rightarrow C(n-1)^{*}$. Now $c_{n-1}(n-1)=w_{2^{n-1}} \bmod H^{*}(B V ; \mathbb{Z} / 2)$ implies $i^{*} c_{n}(n)=c_{n-1}(n-1)^{2}$. Since the order of $c_{n-1}(n)$ is $2^{n-1}$ and the order of $c_{n-1}(n-1)$ is $2^{n}$, we know that $i^{*} c_{n-1}(n)=2^{s} c_{n-1}(n-1)$ for some $s>0$. A corestriction argument now implies $s=1$, since the index of $D(n-1)$ in $D(n)$ is 2 . 
The elements $a$ and $b_{j}$ are natural in the sense that $i^{*}(a)=a$ and $i^{*}\left(b_{j}\right)=b_{j}$ for $1 \leq j \leq n-2$, abusing notation. Thus $i^{*} c_{j}(n)=c_{j}(n-1)$ for $j<n-1$, and we obtain

Corollary 3.2. If $n \geq 2$, there is an additive isomorphism

$$
C(n)^{*} \cong \mathbb{Z}\left\{1,2 \bar{w}_{2^{2}}^{\epsilon_{2}} \cdots \bar{w}_{2^{n-1}}^{\epsilon_{n-1}} \mid \epsilon_{i}=0 \text { or } 1\right\}\left[\bar{w}_{2^{n}}\right] /\left(2^{i+1} \bar{w}_{2^{i}}=0 \mid 2 \leq i \leq n\right),
$$

where the $\bar{w}_{2^{i}}$ are the reductions of the elements $w_{2^{i}}$ in $H^{2^{i}}(B D(i))$.

Remark. When $n=1$, the element $w_{2} \in H^{*}\left(B D_{8} ; \mathbb{Z} / 2\right)$ does not lift to the integral cohomology and $C(1)^{*} \cong \mathbb{Z}\left[\bar{w}_{2}^{2}\right] /\left(4 \bar{w}_{2}^{2}\right)$.

\section{Brown-Peterson cohomology of $B D(n)$}

Let $B P^{*}(-; \mathbb{Z} / 2)$ denote $B P$-theory mod 2 with coefficients

$$
B P^{*} /(2)=\mathbb{Z} / 2\left[v_{1}, v_{2}, \ldots\right] .
$$

We consider the Atiyah-Hirzebruch spectral sequence

$$
E_{2}^{*, *}=H^{*}(B D(n) ; \mathbb{Z} / 2) \otimes B P^{*} \Longrightarrow B P^{*}(B D(n) ; \mathbb{Z} / 2) .
$$

Lemma 4.1. The elements $x_{i}^{2}$ and $w_{2^{n}}^{2}$ are permanent cycles in the spectral sequence (4.1).

Proof. These elements are the top Chern classes of the representations $e_{i \mathbb{C}}$ and $\Delta_{\mathbb{C}}$, respectively.

It is well-known that some of the differentials of (4.1) are given by

$$
d_{2^{i+1}-1}(x)=v_{i} \otimes Q_{i} x \quad \bmod \left(v_{1}, \ldots, v_{i-1}\right) .
$$

Since $Q_{n-1} w_{2^{n}} \neq 0$ by Corollary [2.3, we know that $w_{2^{n}}$ cannot be a permanent cycle, which implies $w_{2^{n}} \notin \operatorname{Im}\left[\rho_{\mathbb{Z} / 2}: B P^{*}(B D(n)) \rightarrow H^{*}(B D(n) ; \mathbb{Z} / 2)\right]$. Thus the integral lift $\bar{w}_{2^{n}}$ of $w_{2^{n}}$ does not lie in the image of $\rho: B P^{*}(B D(n)) \rightarrow H^{*}(B D(n))$, either.

As above, let $\tilde{W}$ denote a maximal elementary abelian subgroup of $D(n)$, and $w(\Delta)$ the total Stiefel-Whitney class of $\Delta$. Then

$$
\begin{aligned}
\operatorname{Res}_{\tilde{W}}^{D(n)}(w(\Delta)) & =\prod(1+x+z)=(1+z)^{2^{n}}+D_{1}(1+z)^{2^{n-1}}+\cdots+D_{n}(1+z) \\
& =1+D_{1}+\cdots+D_{n}+\operatorname{Res}_{\tilde{W}}^{D(n)}\left(w_{2^{n}}\right) ;
\end{aligned}
$$

in particular,

$$
\operatorname{Res}_{\tilde{W}}^{D(n)}\left(w_{2^{n}-2^{n-i}}(\Delta)\right)=D_{i} .
$$

Hence, by (2.2), we can choose polynomials $\tilde{D}_{i} \in \mathbb{Z} / 2\left[x_{1}, \ldots, x_{2 n}\right] \cong H^{*}(B V ; \mathbb{Z} / 2)$ with $w_{2^{n}-2^{n-i}}=\tilde{D}_{i}$. Recall that $J_{V}$ denotes the image of $H^{*}(B V)$ in $H^{*}(B D(n))$.

Theorem 4.2. There is an element in $B P^{*}(B D(n))$,

$$
x=c_{2^{n-1}}\left(\Delta_{\mathbb{C}}\right)-\tilde{D}_{1}\left(c_{1}\left(e_{1 \mathbb{C}}\right), \ldots, c_{1}\left(e_{2 n \mathbb{C}}\right)\right),
$$

which is non-zero in $B P^{*}(B D(n)) \otimes_{B P^{*}} \mathbb{Z} / 2$, and such that

(1) $\rho(x)=2 \bar{w}_{2^{n}} \bmod J_{V}$,

(2) $\rho_{\mathbb{Z} / 2}(x)=0$ in $H^{*}(B D(n) ; \mathbb{Z} / 2)$. 
Proof. Since $x$ is defined via Chern classes, it is an element of $B P^{*}(B D(n))$. Assertion (2) is immediate from (4.3). Since $\bar{w}_{2^{n}} \notin \operatorname{Im}(\rho)$, to prove (1) it suffices to show that $x$ is a $B P^{*}$-module generator. Let $F=\left\langle a_{1} a_{2}\right\rangle \subset D(n)$; this is cyclic of order 4 . By the double coset formula,

$$
\begin{aligned}
\operatorname{Res}_{F}^{D(n)} \operatorname{Ind}_{\tilde{V}^{\text {odd }}}^{D(n)}\left(e_{\mathbb{C}}\right) & =\bigoplus_{F g \tilde{V}^{\text {odd }}} \operatorname{Ind}_{F \cap g^{-1} \tilde{V}^{\text {odd } g}}^{F} \operatorname{Res}_{F \cap g^{-1} \tilde{V}^{\text {odd } g}}^{g^{-1} \tilde{V}^{\text {odd } g}}\left(g^{*} e_{\mathbb{C}}\right) \\
& =\bigoplus \operatorname{Ind}_{N}^{2^{n-1}}\left(e_{\mathbb{C}}\right),
\end{aligned}
$$

since the elements $g=a_{2}^{\epsilon_{2}} \cdots a_{2 n}^{\epsilon_{2 n}}, \epsilon_{i}=0$ or 1 , form a complete set of double coset representatives. Notice that $\operatorname{Ind}_{N}^{F}\left(e_{\mathbb{C}}\right)$ decomposes as $e_{F} \oplus-e_{F}$, where $e_{F}$ is a faithful 1-dimensional complex representation of $\mathbb{Z} / 4$. Thus the total Chern class of $\Delta_{\mathbb{C}}$ restricts to $F$ as

$$
\operatorname{Res}_{F}\left(c\left(\Delta_{\mathbb{C}}\right)\right)=((1+u)(1-u))^{2^{n-1}}=\left(1-u^{2}\right)^{2^{n-1}} \quad \text { with } H^{*}(B F) \cong \mathbb{Z}[u] /(4 u) .
$$

Consequently, we have $\operatorname{Res}_{F}\left(c_{2^{n-1}}\left(\Delta_{\mathbb{C}}\right)\right)=2 u^{2^{n-1}}$ in $H^{*}(F)$. Since $\operatorname{Res}_{F}\left(c_{1}\left(e_{i \mathbb{C}}\right)\right)=$ $2 \lambda_{i} u$ for some $\lambda_{i} \in \mathbb{Z} / 4$, we immediately obtain $\operatorname{Res}_{F}\left(\tilde{D}_{i}\right)=0$, and therefore (1).

Now recall the following lemma of Totaro.

Lemma 4.3 ([T1]). Let $p$ be a prime and $X$ any space. If $\rho_{\mathbb{Z} / p}: B P^{*}(X) \otimes_{B P^{*}}$ $\mathbb{Z}_{(p)} \rightarrow H^{*}(X ; \mathbb{Z} / p)$ is not injective, then $\rho: B P^{*+2}(X \times B \mathbb{Z} / p) \otimes_{B P^{*}} \mathbb{Z}_{(p)} \rightarrow$ $H^{*+2}(X \times B \mathbb{Z} / p)$ is also not injective.

Proof. We have $B P^{*}(B \mathbb{Z} / p) \otimes_{B P^{*}} \mathbb{Z}_{(p)} \cong H^{*}(B \mathbb{Z} / p)_{(p)} \cong \mathbb{Z}_{(p)}[u] /(p u)$ with $u$ in degree two. If $\rho_{\mathbb{Z} / p}(x)=0$, then $\rho(x \otimes u)=0$ in $H^{*}(X \times B \mathbb{Z} / p)_{(p)}$. On the other hand, it is well-known that $B P^{*}(B \mathbb{Z} / p)$ is $B P^{*}$-flat and thus $B P^{*}(X \times B \mathbb{Z} / p) \cong$ $B P^{*}(X) \otimes_{B P^{*}} B P^{*}(B \mathbb{Z} / p)$. Hence if $0 \neq x \in B P^{*}(X)_{B P^{*}} \mathbb{Z}_{(p)}$, then $x \otimes u$ is also non-zero in $B P^{*}(X \times B \mathbb{Z} / p) \otimes_{B P^{*}} \mathbb{Z}_{(p)}$.

Let $\rho^{\prime}: C H^{*}(-) \rightarrow H^{*}(-)$ denote the cycle map, and $\rho_{\mathbb{Z} / 2}^{\prime}$ the cycle map followed by reduction modulo 2 . Since Chow rings have Chern classes, we easily deduce

Corollary 4.4. There is a non-zero element $x^{\prime}$ in $C H^{2^{n}}(B D(n))$ satisfying

(1) $\rho^{\prime}\left(x^{\prime}\right)=2 \bar{w}_{2^{n}} \bmod J_{V}$;

(2) $\rho_{\mathbb{Z} / 2}^{\prime}\left(x^{\prime}\right)=0$.

Hence $\rho^{\prime}: C H^{2^{n}+2}(B(D(n) \times \mathbb{Z} / 2)) \rightarrow H^{2^{n}+2}(B(D(n) \times \mathbb{Z} / 2))$ is not injective.

Remark. First note that the above argument does not hold for $n=1$. Indeed, in that case $H^{*}\left(B D_{8}\right) \subset \operatorname{Im}(\rho)$ modulo $H^{*}(B V)$. Similar facts hold for 2-groups $G$ which have a cyclic maximal normal subgroup [S], i.e. dihedral, semidihedral, quasidihedral, and generalized quaternion groups of order a power of 2. Moreover, $B P^{*}(B G)$ is generated by Chern classes for these groups. The extraspecial 2-groups of order $2^{2 n+1}$ are of two types. Quillen calls them the real and the quaternionic type, where the real type corresonds to the groups $D(n)$ considered above, and the quaternionic group of order $2^{n+1}$ is the central product of $D(n-1)$ with the quaternion group $Q_{8}$ of order 8 . Consider now this second case, and denote this group by $D^{\prime}(n)$; it also has center $\mathbb{Z} / 2$ with quotient $V \cong(\mathbb{Z} / 2)^{2 n}$. In Quillen's 
notation $\mathrm{Q}$, this corresponds to $h=n+1$ and $r=2$. The quadratic form (extension class) is $f=x_{1}^{2}+x_{1} x_{2}+x_{2}^{2}+\sum_{i=2}^{n} x_{2 i-1} x_{2 i}$, and the cohomology is given by

$$
H^{*}\left(B D^{\prime}(n) ; \mathbb{Z} / 2\right) \cong \mathbb{Z} / 2\left[w_{2^{n+1}}\right] \otimes \mathbb{Z} / 2\left[x_{1} \ldots, x_{2 n}\right] /\left(f, Q_{0} f, \ldots, Q_{n-1} f\right) .
$$

Here the $x_{i}$ are as before the generators of $H^{*}(B V ; \mathbb{Z} / 2)$ inflated to $D^{\prime}(n)$, and $w_{2^{n+1}}$ is the Euler class of the $2^{n+1}$-dimensional irreducible representation $\Delta$. The cohomology of $D^{\prime}(n)$ is also detected on subgroups $\tilde{W} \cong Q_{8} \times W$ in one-to-one correspondence with maximal isotropic subspaces, i.e. there is an injection

$$
H^{*}\left(B D^{\prime}(n) ; \mathbb{Z} / 2\right) \hookrightarrow \prod_{W} H^{*}\left(B\left(Q_{8} \times W\right) ; \mathbb{Z} / 2\right),
$$

where $W$ ranges over the maximal isotropic subspaces of $V$ (which have dimension $n-1)$. The Stiefel-Whitney classes $w_{j}(\Delta)$ are zero except for the following values of $j([\mathrm{Q}],(5.6))$ :

$\operatorname{Res}_{Q_{8} \times W}\left(w_{j}(\Delta)\right)= \begin{cases}\left(D_{i}^{\prime}\right)^{4} & \text { for } j=2^{h}-2^{h-i}, 1 \leq i \leq n-1, \\ \sum_{i=0}^{n-2} e^{2^{i}}\left(D_{n-i-1}^{\prime}\right)^{4} & \text { for } j=2^{n+1},\end{cases}$

where $e \in H^{4}\left(Q_{8} ; \mathbb{Z} / 2\right)$ is the Euler class of the obvious 4-dimensional irreducible representation of $Q_{8}$, and $D_{i}^{\prime}$ is the degree $\left(2^{n-1}-2^{n-1-i}\right)$ Dickson invariant for rank $n-1$. Thus almost all arguments for $D(n)$ work in this case, too, except for $Q_{m} w_{j}(\Delta)=0$. For example, we can define $x=c_{2^{n}}\left(e_{\mathbb{C}}\right)-\left(\tilde{D}_{1}^{\prime}\right)^{4}$ in $B P^{*}\left(B D^{\prime}(n)\right)$; this class satisfies $\rho(x)=2 \bar{w}_{2^{n+1}}$ and $\rho_{\mathbb{Z} / 2}(x)=0$. However, it seems that we cannot prove that $x$ is a $B P^{*}$-module generator of $B P^{*}\left(B D^{\prime}(n)\right)$, because $\operatorname{Res}_{N}\left(c_{2^{n}}\left(\operatorname{Ind}_{\mathbb{Z} / 4 \oplus W}^{D^{\prime}(n)}\left(e_{F}\right)\right)\right)=u^{2^{n}}$ and $w_{2^{n+1}}(\Delta) \in \operatorname{Im}(\rho) \bmod \left(H^{*}(B V)\right)$.

\section{Permanent cycles}

This section deals with the Atiyah-Hirzebruch spectral sequence converging to $B P^{*}(B D(n))$. In the course of the section, we shall make several technical assumptions on the behaviour of this spectral sequence. These will be verified for $n=3,4$ in Section 7.

Given a space $X$, each non-zero element $x \in B P^{*}(X)$ with $\rho(x)=0 \in H^{*}(X)_{(p)}$ is represented by a non-zero element in $E_{\infty}^{*, a}$ with $a<0$ in the Atiyah-Hirzebruch spectral sequence converging to $B P^{*}(X)$.

Assumption 5.1. Let $n \geq 3$. In the Atiyah-Hirzebruch spectral sequence converging to $B P^{*}(B D(n))$, every nonzero element in the ideal $\left(2, v_{1}, \ldots, v_{n-2}\right) \otimes \bar{w}_{2^{n}}$ is a nonzero permanent cycle.

The outer automorphism group of $D(n)$ is the orthogonal group $O(V)$ of $V$ associated to the quadratic form $q([\mathrm{BC}]$, p. 216$)$. Since $\Delta$ is the unique irreducible representation which acts non-trivially on the center, the element $w_{2^{n}}$ is invariant under the orthogonal group ( $\mathrm{Q}$, Remark 4.7). Moreover, the invariant ring generated by the Stiefel-Whitney classes of $\Delta([\mathbf{Q})$, Corollary 5.12 and Remark $5.14)$,

$$
H^{*}(B D(n) ; \mathbb{Z} / 2)^{O(V)}=\mathbb{Z} / 2\left[\tilde{D}_{1}, \ldots, \tilde{D}_{n}, w_{2^{n}}\right] \text { with } \tilde{D}_{i}=w_{2^{n}-2^{n-i}}(\Delta) .
$$

To consider the Atiyah-Hirzebruch spectral sequence

$$
E_{2}^{*, *}(X)=H^{*}(X) \otimes B P^{*} \Longrightarrow B P^{*}(X)
$$


for the spaces $X=B D(n)$ or $B \operatorname{Spin}(m)$, we need the integral version of the above invariant ring. Note that $\beta\left(\tilde{D}_{n-1}\right)=\tilde{D}_{n}$; let

$$
W(\Delta)=\mathbb{Z}_{(2)}\left[\tilde{D}_{1}, \ldots, \tilde{D}_{n-2}, \tilde{D}_{n-1}^{2}, \tilde{D}_{n}, w_{2^{n}}\right] /\left(2 \tilde{D}_{n}\right) .
$$

Suppose $X$ is a space such that

$$
\begin{aligned}
& \text { there is a map } f: W(\Delta) \rightarrow H^{*}(X)_{(2)} \text { such that } \\
& W(\Delta) /(2) \subset H^{*}(X ; \mathbb{Z} / 2) \text { as } \Lambda\left(Q_{0}, \ldots, Q_{n}\right) \text {-algebras. }
\end{aligned}
$$

In Section 7 we shall see that we may take $X=B \operatorname{Spin}(7)$ and $X=B \operatorname{Spin}(9)$ for the cases $n=3$ and $n=4$, respectively. In the spectral sequence for $B D(n)$, let $W(\Delta)_{r}$ be the subalgebra of $E_{r}^{*, *}$ which is the subquotient algebra of $B P^{*} \otimes$ $f(W(\Delta))$. In general, the invariants $\left(E_{r}^{*, *}\right)^{O(V)}$ are not equal to $W(\Delta)_{r}$. Below we consider the case were $W(\Delta)_{r}$ is nevertheless closed under the differentials.

Lemma 5.2. Let $n \geq 3$. Suppose that $X$ satisfies (5.2) and $d_{r}\left(W(\Delta)_{r}\right) \subset W(\Delta)_{r}$ for all $r \geq 2$ in the spectral sequence (5.1). Then each element in the ideal $\left(2, v_{1}, \ldots, v_{n-2}\right) \otimes w_{2^{n}}$ and the ideal $\left(2, v_{1}, \ldots, v_{n-2-i}\right) \otimes \tilde{D}_{i}, 1 \leq i \leq n-2$, is a permanent cycle. Moreover, if $w_{2^{n}}\left(\right.$ resp. $\left.\tilde{D}_{i}\right)$ is not in the image of $Q_{k}$, then $\left[v_{k} \otimes w_{2^{n}}\right]\left(r e s p .\left[v_{k} \otimes \tilde{D}_{i}\right], i \leq n-k-2\right)$ is a non-zero element in $E_{\infty}^{* * *}$.

Before beginning with the proof, recall the cohomology theory $P(m)^{*}(-)$ with coefficients $B P^{*} /\left(2, v_{1}, \ldots, v_{m-1}\right) \cong \mathbb{Z} / 2\left[v_{m}, v_{m+1}, \ldots\right]$. In particular, the theory $P(1)^{*}(-)$ is $\bmod 2 B P$-theory $B P^{*}(-; \mathbb{Z} / 2)$.

Proof. First note that $\left|\tilde{D}_{i}\right|=2^{n}-2^{n-i}$, which is even except for the case $i=n$. Hence

$$
W(\Delta)_{2}^{\text {odd }}=P(1)^{*}\left[w_{2^{n}}, \tilde{D}_{1}, \ldots, \tilde{D}_{n-2}, \tilde{D}_{n-1}^{2}, \tilde{D}_{n}^{2}\right]\left\{\tilde{D}_{n}\right\} .
$$

By induction, we assume that for $2^{r} \leq i \leq 2^{r+1}-1$

$$
\begin{gathered}
W(\Delta)_{i}^{\text {odd }}=P(r)^{*} \otimes A \otimes B_{r} \otimes C_{r}\left\{\tilde{D}_{n}\right\} \quad \text { with } \\
A=\mathbb{Z} / 2\left[w_{2^{n}}\right], B_{r}=\mathbb{Z} / 2\left[\tilde{D}_{1}, \ldots, \tilde{D}_{n-r-1}\right], C_{r}=\mathbb{Z} / 2\left[\tilde{D}_{n-r}^{2}, \ldots, \tilde{D}_{n}^{2}\right] .
\end{gathered}
$$

Let $E(P(m))_{i}^{*, *}$ denote the Atiyah-Hirzebruch spectral sequence converging to $P(m)^{*}(X)$, and let $\rho_{i}: E_{i}^{*, *} \rightarrow E(P(m))_{i}^{*, *}$ be the map of spectral sequences induced from the natual transformation $\rho: B P^{*}(X) \rightarrow P(m)^{*}(X)$ of cohomology theories. Since $\left|v_{r}\right|=-2^{r+1}+2$, we see that $E(P(r))_{2^{r+1}-1}^{* * *} \cong E(P(r))_{2}^{*, *}$ for degree reasons. Now $W(\Delta)_{i}^{\text {odd }}$ is $P(r)^{*}$-free, so the restriction map $\rho_{i} \mid W(\Delta)_{i}$ is injective for $i<2^{r+1}$. Hence there is no element $x$ with $0 \neq d_{i}(x) \in W(\Delta)_{i}^{o d d}$, and we have $W(\Delta)_{2^{r}}^{o d d} \cong W(\Delta)_{2^{r+1}-1}^{o d d}$. Except for $\tilde{D}_{n-r-1}$, generators in $W(\Delta)_{i}$ are annihilated by $Q_{r}$ and thus by $d_{2^{r+1}-1}$. The non-zero differential is

$$
d_{2^{r+1}-1}\left(\tilde{D}_{n-r-1}\right)=v_{r} \otimes Q_{r}\left(\tilde{D}_{n-r-1}\right)=v_{r} \otimes \tilde{D}_{n} .
$$

Therefore (5.3) also holds for $i=2^{r+1}$. Since $W(\Delta)_{i}$ is a $P(r)^{*}$-module, every element in the ideal $\left(2, \ldots, v_{r-1}\right) \otimes \tilde{D}_{n-r-1}$ is a cycle in $E_{2^{r+1}}^{* * *}$. Consequently we get

$$
W(\Delta)_{2^{n}-1}^{\text {odd }}=P(n-1)^{*} \otimes A \otimes C_{n-1}\left\{\tilde{D}_{n}\right\} .
$$

The next differential is

$$
d_{2^{n}-1}\left(w_{2^{n}}\right)=v_{n-1} \otimes w_{2^{n}} \tilde{D}_{n} \quad \text { and } \quad d_{2^{n}-1}\left(\tilde{D}_{n}\right)=v_{n-1} \otimes \tilde{D}_{n}^{2} .
$$


Hence we have

$$
W(\Delta)_{2^{n+1}-1}^{o d d}=P(n)^{*} \otimes C_{n}\left\{\tilde{D}_{n} w_{2^{n}}\right\}, \quad \text { where } \quad C_{n}=\mathbb{Z} / 2\left[w_{2^{n}}^{2}\right] \otimes C_{n-1} .
$$

Here we note that each element in the ideal $\left(2, v_{1}, \ldots, v_{n-2}\right) \otimes w_{2^{n}}$ is a cycle in $E_{2^{n}}^{* * *}$, because $w_{2^{n}} \tilde{D}_{n}$ generates a $P(n-1)^{*}$-module in $E_{2^{n}}^{* *}$.

Finally, we consider the differential

$$
d_{2^{n+1}-1}\left(w_{2^{n}} \tilde{D}_{n}\right)=v_{n} \otimes Q_{n+1}\left(w_{2^{n}} \tilde{D}_{n}\right)=v_{n} \otimes\left(w_{2^{n}}^{2} \tilde{D}_{n}^{2}\right) .
$$

Thus $W(\Delta)_{2^{n+1}}^{o d d}=0$, and each element in the above ideals is a permanent cycle. If $w_{2^{r}} \notin \operatorname{Im}\left(Q_{r}\right)$, then, considering the map $\rho_{2^{r}-1}$, we see that $\left[v_{r} \otimes w_{2^{n}}\right]$ is nonzero.

Next we consider the mod 2 version of the above arguments. We study the Atiyah-Hirzebruch spectral sequence

$$
E_{2}=H^{*}(X ; \mathbb{Z} / 2) \otimes P(1)^{*} \Longrightarrow P(1)^{*}(X) .
$$

Denote the invariant ring by

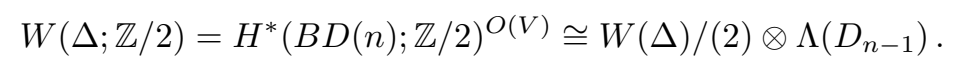

We consider the following situation:

(5.5) there is an injection $W(\Delta ; \mathbb{Z} / 2) \subset H^{*}(X ; \mathbb{Z} / 2)$ as $\Lambda\left(Q_{0}, \ldots Q_{n+1}\right)$-algebras.

In the spectral sequence (5.4), let $W(\Delta ; \mathbb{Z} / 2)_{r}$ be the subalgebra of $E_{r}^{*, *}$ which is the subquotient algebra of $P(1)^{*} \otimes(W(\Delta) ; \mathbb{Z} / 2)$. Of course the mod 2 reductions of the permanent cycles in (5.1) are also permanent cycles in (5.4). Moreover we have

Lemma 5.3. Let $n \geq 3$. Suppose that $X$ satisfies (5.5) and $d_{r}\left(W(\Delta ; \mathbb{Z} / 2)_{r}\right) \subset$ $W(\Delta ; \mathbb{Z} / 2)_{r}$ for all $r \geq 2$ in the spectral sequence (5.4). Then every element in the ideal $\left(v_{1}, \ldots, v_{n-1}\right) \otimes w_{2^{n}} \tilde{D}_{n-1}$ or $\left(v_{1}, \ldots, v_{n-2}\right) \otimes \tilde{D}_{n-1}$ is a permanent cycle.

Proof. The proof is similar to the $B P^{*}$-case. In particular, for $i \leq 2^{n}-1$, we have $W(\Delta ; \mathbb{Z} / 2)_{i}^{\text {odd }}=W(\Delta)_{i}^{\text {odd }} /(2) \otimes \Lambda\left(D_{n-1}\right)$. The difference starts with

$$
d_{2^{n}-1}(x)=v_{n-1} \otimes \tilde{D}_{n} x \quad \text { for } x=w_{2^{n}}, \tilde{D}_{n-1}, \tilde{D}_{n} .
$$

Hence we get

$$
W(\Delta ; \mathbb{Z} / 2)_{2^{n+1}-1}^{o d d}=P(n)^{*} \otimes C_{n}\left\{\tilde{D}_{n} w_{2^{n}}, \tilde{D}_{n} \tilde{D}_{n-1}\right\} .
$$

Here note that $\tilde{D}_{n-1} w_{2^{n}}$ is a cycle in $E_{2^{n+1}-1}^{*, *}$. We also know that each element in the ideal $\left(v_{1}, \ldots, v_{n-2}\right) \otimes \tilde{D}_{n-1}$ is a cycle in $E_{2^{n+1}-1}^{*, *}$.

From Lemma 2.4 and (2.3), the image of $w_{2^{n}} \tilde{D}_{n}$ (resp. $\tilde{D}_{n-1} \tilde{D}_{n}$ ) under the differential $d_{2^{n+1}}$ is $v_{n} \otimes\left(w_{2^{n}}^{2} \tilde{D}_{n}^{2}\right)$ (resp. $\left.\tilde{D}_{n}^{4}\right)$. Hence we see that

$$
\operatorname{Ker}\left(W(\Delta ; \mathbb{Z} / 2)_{2^{n+1}-1}^{\text {odd }}\right)=P(n)^{*} \otimes C_{n}\{a\} \quad \text { where } a=\tilde{D}_{n-1} \tilde{D}_{n} w_{2^{n}}^{2}+\tilde{D}_{n}^{3} w_{2^{n}} .
$$

Since $d_{2^{n+1}-1}\left(w_{2^{n}} \tilde{D}_{n-1}\right)=v_{n} \otimes a$, we get $W(\Delta ; \mathbb{Z} / 2)_{2^{n+2}-1}^{\text {odd }}=P(n+1)^{*} \otimes C_{n}\{a\}$. Here note that $v_{n-1} \otimes w_{2^{n}} \tilde{D}_{n}$ is a cycle in $E_{2^{n+2}-1}^{o d d}$. The last nonzero differential is, again by Lemma 2.4 ,

$$
d_{2^{n+2}-1}(a)=v_{n+1} \otimes \tilde{D}_{n}^{4} w_{2^{n}}^{4} .
$$

Thus $W(\Delta ; \mathbb{Z} / 2)_{2^{n+2}}^{o d d}=0$. Hence we get the permanency of elements in the lemma. 
If $B P^{*}(X)$ is 2-torsion free, e.g., $B P^{*}(X)$ and $P(1)^{*}(X)$ are generated by even dimensional elements, then the Bockstein exact sequence induces an isomorphism $B P^{*}(X) /(2) \cong P(1)^{*}(X)$. In particular, $B P^{*}(X) \otimes_{B P^{*}} \mathbb{Z} / 2 \cong P(1)^{*}(X) \otimes_{P(1)^{*}} \mathbb{Z} / 2$. These facts hold for $X=B \operatorname{Spin}(m)$ for $m=7,9$ (see Section 7 below). The following assertion seems reasonable for dimensional reasons.

Assumption 5.4. Suppose that (5.2) holds and $B P^{*}(X) /(2) \cong P(1)^{*}(X)$. The

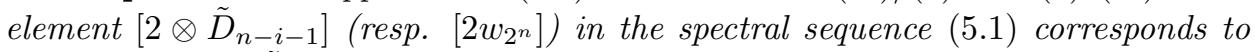
the element $\left[v_{i} \otimes \tilde{D}_{n-1}\right]$ (resp. $\left.\left[v_{n-1} \otimes w_{2^{n}} D_{n-1}\right]\right)$ in the spectral sequence (5.4) $)$ e.g. the element $x$ with $\rho_{\mathbb{Z} / 2}(x)=0$ in Theorem 4.2 is represented by $\left[v_{n-1} \otimes w_{2^{n}} D_{n-1}\right]$.

Remark. Let $M_{p}$ be the Moore space such that $H^{2}\left(M_{p}\right) \cong \mathbb{Z} / p$. Then there is an isomorphism $P(1)^{*}(X) \cong B P^{*+2}\left(X \wedge M_{p}\right)$ if $B P^{*}(X)$ is $p$-torsion free. Hence we can deduce the behaviour of the Atiyah-Hirzebruch spectral sequence converging to $B P^{*}(D(n) \times B \mathbb{Z} / 2)$ from that converging to $P(1)^{*}(B D(n))$.

\section{Transfers of Chern Classes}

To study Chern classes, we consider the restriction to the center $N \cong \mathbb{Z} / 2$ of $D(n)$. Let $I$ denote the ideal $\left(2, v_{1}, v_{2}, \ldots\right)$ in $B P^{*}$. Then

$$
\rho_{\mathbb{Z} / 2}: B P^{*}(B N) / I \cong \mathbb{Z} / 2\left[z^{2}\right] \subset H^{*}(B N ; \mathbb{Z} / 2) .
$$

Since the image of the restriction $H^{*}(B D(n) ; \mathbb{Z} / 2) \rightarrow H^{*}(B N ; \mathbb{Z} / 2)$ is generated by $w_{2^{n}} \notin \operatorname{Im}\left(\rho_{\mathbb{Z} / 2}\right)$, we see that

$$
\operatorname{Im}\left[B P^{*}(B D(n)) \rightarrow B P^{*}(B N) / I\right]=\mathbb{Z} / 2\left[u^{2^{n}}\right],
$$

where $u$ denotes the obvious generator in degree 2 . Let $\xi$ be a complex representation of $D(n)$; it restricts to $N$ as the sum of $m$ copies (say) of the nontrivial character $e_{\mathbb{C}}$ plus some trivial representations. Then there is an element $u^{\prime} \equiv u$ $\bmod I$ in $B P^{*}(B N)$ with

$$
\operatorname{Res}_{N}(c(\xi))=\left(1+u^{\prime}\right)^{m},
$$

where $c(\xi)$ denotes as usual the total Chern class of $\xi$. Then $u^{m}$ lies in the image of $B P^{*}(B D(n)) \rightarrow B P^{*}(B N) / I$, and so $m$ has to be divisible by $2^{n}$.

Proposition 6.1. Suppose Assumption 5.1 holds and $n \geq 3$. Then the permanent cycles $\left[v_{1} \bar{w}_{2^{n}}\right], \ldots,\left[v_{n-1} \bar{w}_{2^{n}}\right]$ are not represented by $B P^{*}$-linear combinations of products of Chern classes.

Proof. Let $\xi$ be a representation satifying (6.2) for some $m=2^{n} m^{\prime}$. The restriction of the total Chern class of $\xi$ is given by

$$
\begin{aligned}
\operatorname{Res}_{N}(c(\xi)) & =1+2 m^{\prime}\left(u^{\prime}\right)^{2^{n-1}} \bmod \left(I^{2}, u^{2^{n}}\right) \\
& =1+m^{\prime}\left(v_{1} u^{2^{n-1}+1}+\cdots+v_{i} u^{2^{n-1}+2^{i}-1}+\cdots\right) \bmod \left(I^{2}, u^{2^{n}}\right),
\end{aligned}
$$

which does not contain the term $v_{i} u^{2^{n-1}}$. But

$$
\operatorname{Res}_{N}\left(\left[v_{i} \bar{w}_{2^{n}}\right]\right)=v_{i} u^{2^{n-1}} \bmod \left(u^{2^{n-1}+1}\right) .
$$

Hence no $B P^{*}$-linear combination of products of Chern classes can represent $\left[v_{i} \bar{w}_{2^{n}}\right]$.

Theorem 6.2. Suppose Assumption 5.1 holds and $n \geq 3$. Then the permanent cycles $\left[v_{1} \bar{w}_{2^{n}}\right], \ldots,\left[v_{n-2} \bar{w}_{2^{n}}\right]$ are not represented by transfers of $B P^{*}$-linear combinations of products of Chern classes. 
Proof. Let $H$ be a subgroup of $D(n)$, and suppose $\left[v_{j} \bar{w}_{2^{n}}\right]=\operatorname{Tr}_{H}^{D(n)}(x)$ for some $x \in B P^{*}(B H)$. By the double coset formula,

$$
\operatorname{Res}_{N}^{D(n)} \operatorname{Tr}_{H}^{D(n)}(x)=\sum_{H g N} \operatorname{Tr}_{g^{-1} H g \cap N}^{N} \operatorname{Res}_{g^{-1} H g \cap N}^{g^{-1} H g}\left(g^{*} x\right),
$$

where the sum ranges over double coset representatives $g$ of $H \backslash D(n) / N$. If $H$ intersects $N$ trivially, then so does any conjugate of $H$. Hence we need only consider subgroups $H$ containing the center, and the double coset formula evaluates to $|D(n) / H| \cdot \operatorname{Res}_{N}(x)$. Since this element is represented by

$$
\operatorname{Res}_{N}\left[v_{i} \bar{w}_{2^{n}}\right]=v_{i} u^{2^{n-1}} \not \equiv 0 \bmod I^{2},
$$

we get $|D(n) / H|=2$, and thus $H \cong D(n-1) \times \mathbb{Z} / 2$ or $H \cong D(n-1) \times_{N} \mathbb{Z} / 4$.

The total Chern class $c(\zeta)$ of any representation $\zeta$ of $D(n-1)$ restricts as

$$
\operatorname{Res}_{N}(c(\zeta))=\left(1+u^{\prime}\right)^{2^{n-1} m}=1+m u^{2^{n-1}} \bmod \left(I, u^{2^{n}}\right) .
$$

Hence we have

$$
\begin{aligned}
\operatorname{Res}_{N}(2 c(\zeta))= & 2+2 m u^{2^{n-1}}=\left(v_{1} u^{2}+\cdots+v_{i} u^{2^{i}}+\cdots\right) \\
& +m\left(v_{1} u^{2^{n-1}+1}+\cdots+v_{i} u^{2^{n-1}+2^{i-1}}+\cdots\right) \bmod \left(I^{2}, u^{2^{n}}\right),
\end{aligned}
$$

which does not contain $v_{i} u^{2^{n-1}}$. Thus $\left[v_{j} \bar{w}_{2^{n}}\right]$ is not represented by any $B P^{*}$-linear combination of products of Chern classes.

$$
\text { 7. } B P^{*}(B \operatorname{Spin}(7)) \text { AND } B P^{*}(B \operatorname{Spin}(9))
$$

The mod 2 cohomology of $B \operatorname{Spin}(n)$ was computed by Quillen [Q]:

$$
H^{*}(B \operatorname{Spin}(n) ; \mathbb{Z} / 2) \cong \mathbb{Z} / 2\left[w_{2^{h}}(\Delta)\right] \otimes \mathbb{Z} / 2\left[w_{2}, \ldots, w_{n}\right] /\left(w_{2}, Q_{0} w_{2}, \ldots, Q_{h-1} w_{2}\right),
$$

where $\Delta$ is a spin representation of $\operatorname{Spin}(n)$ and $2^{h}$ the Radon-Hurwitz number (see Q, $\S 6)$. This is proved by calculating the Serre spectral sequence of the fibration

$$
B \mathbb{Z} / 2 \longrightarrow B \operatorname{Spin}(n) \longrightarrow B S O(n) \text {. }
$$

We consider the case $n=7$. Then $h=3$, and the $\bmod 2$ cohomology of $B \operatorname{Spin}(n)$ is a polynomial algebra on the Stiefel-Whitney classes $w_{4}, w_{6}, w_{7}, w_{8}$ of a spin representation, i.e.

$$
H^{*}(B \operatorname{Spin}(7) ; \mathbb{Z} / 2) \cong \mathbb{Z} / 2\left[w_{4}, w_{6}, w_{7}, w_{8}\right] .
$$

Recall that $\operatorname{Spin}(7)$ has the exceptional Lie group $G_{2}$ as a subgroup. $G_{2}$ contains a rank three elementary abelian 2-subgroup, and its mod 2 cohomology is isomorphic to the rank three Dickson invariants, i.e. $H^{*}\left(B G_{2} ; \mathbb{Z} / 2\right) \cong \mathbb{Z} / 2\left[D_{1}, D_{2}, D_{3}\right]$. Here we may identify the Dickson invariants with the Stiefel-Whitney classes of the restriction of the spin representation to $G_{2}$, namely $D_{1}=w_{4}, D_{2}=w_{6}$, and $D_{3}=w_{7}$. In particular, we have $H^{*}(B \operatorname{Spin}(7) ; \mathbb{Z} / 2) \cong \mathbb{Z} / 2\left[D_{1}, D_{2}, D_{3}\right] \otimes \mathbb{Z} / 2\left[w_{8}\right]$.

Thus $H^{*}(B \operatorname{Spin}(7))=W(\Delta)$, and the technical assumptions of Section 5 are satified with $X=B \operatorname{Spin}(7))$. Hence all results from that section hold in this case.

Indeed, the Brown-Peterson cohomology of $B \operatorname{Spin}(7)$ is given in $[\mathrm{KY}]$. In the Atiyah-Hirzebruch spectral sequence converging to $B P^{*}(B \operatorname{Spin}(7))$, all non-zero differentials are of the form $d_{2^{m}-1}=v_{m-1} \otimes Q_{m-1}$ :

$$
d_{3} w_{4}=v_{1} w_{7}, \quad d_{7} w_{7}=v_{2} w_{7}^{2}, \quad d_{7} w_{8}=v_{2} w_{7} w_{8}, \quad d_{15}\left(w_{7} w_{8}\right)=v_{3} w_{7}^{2} w_{8}^{2} .
$$


Thus

$$
\begin{aligned}
& E_{\infty}^{*, *}=E_{16}^{*, *} \\
& \cong B P^{*}\left\{1,2 w_{4}, 2 w_{8}, 2 w_{4} w_{8}, v_{1} w_{8}\right\} \otimes A \oplus\left(P(3)^{*}\left[w_{7}^{2}\right]\left\{w_{7}^{2}\right\} \otimes A\right) /\left(v_{3} w_{7}^{2} w_{8}^{2}\right) \\
& \quad \text { with } A=\mathbb{Z}_{(2)}\left[w_{4}^{2}, w_{6}^{2}, w_{8}^{2}\right] .
\end{aligned}
$$

For the spectral sequence converging to $P(1)^{*}(B \operatorname{Spin}(7))$, the arguments from Section 5 give

$$
\begin{aligned}
E_{\infty}^{*, *}=E_{32}^{*, *} \cong & P(1)^{*}\left\{1, v_{1} w_{6}, v_{1} w_{8}, v_{1} w_{6} w_{8}, v_{2} w_{6} w_{8}\right\} \otimes A \\
& \oplus P(3)^{*}\left[w_{7}^{2}\right]\left\{w_{7}^{2}\right\} \otimes A /\left(v_{3} w_{7}^{4}, v_{3} w_{7}^{2} w_{8}^{2}, v_{4} w_{7}^{4} w_{8}^{4}\right) .
\end{aligned}
$$

Note that $B P^{*}(B \operatorname{Spin}(7)) /(2)$ is isomorphic to $P(1)^{*}(B \operatorname{Spin}(7))$, which is also implied by the relations

$$
2\left[w_{7}^{2}\right]+v_{3}\left[w_{7}^{4}\right]+\cdots=0 \text { and } 2\left[w_{7}^{2} w_{8}^{2}\right]+v_{4}\left[w_{7}^{4} w_{8}^{4}\right]+\cdots=0,
$$

which follow from the fact that if $\sum v_{i} x_{i}=0$ in $B P^{*}(X)$, then there exist classes $y \in H^{*}(X ; \mathbb{Z} / p)$ with $\rho_{\mathbb{Z} / p}\left(x_{i}\right)=Q_{i} y([\overline{\mathrm{Y} 1}])$.

Theorem 7.1. The element $\left[v_{1} w_{8}\right]$ is not represented by a transfer of a $B P^{*}$-linear combination of products of Chern classes.

Proof. This follows from Proposition 6.1 by looking at the commutative diagram

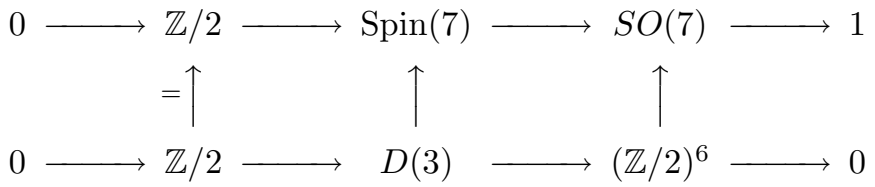

whose rows are central extensions.

Similar arguments work for $\operatorname{Spin}(9)$ and $D(4)$; in this case the Radon-Hurwitz number is 16 . The mod 2 cohomology is

$$
H^{*}(B \operatorname{Spin}(9) ; \mathbb{Z} / 2) \cong H^{*}(B \operatorname{Spin}(7) ; \mathbb{Z} / 2) \otimes \mathbb{Z} / 2\left[w_{16}\right] .
$$

Since $D(4) \subset \operatorname{Spin}(9)$, the above cohomology ring contains the rank four Dickson algebra $\mathbb{Z} / 2\left[D_{1}, \ldots, D_{4}\right]$. The invariant $D_{1}$ is equal to $w_{8}+w_{4}^{2}$, from (2.5). Since $S q^{4} D_{1}=D_{2}$, we get $D_{2}=w_{8} w_{4}+w_{6}^{2}$. Similarly $D_{3}=w_{8} w_{6}+w_{7}^{2}$ and $D_{4}=w_{8} w_{7}$. We consider the Atiyah-Hirzebruch spectral sequence converging to $P(1)^{*}(B \operatorname{Spin}(9))$. The odd degree part of $E_{2}^{*, *}$-page is additively

$$
E_{2}^{* \text { odd }} \cong P(1)^{*}\left[w_{16}\right] \otimes B \otimes \Lambda\left(w_{4}, w_{6}, w_{8}\right)\left\{w_{7}\right\} \quad \text { with } B=\mathbb{Z} / 2\left[w_{4}^{2}, w_{6}^{2}, w_{7}^{2}, w_{8}^{2}\right] .
$$

Using the calculations in Section 5, we can compute

$$
\begin{aligned}
& E_{4}^{* \text { odd }} \cong P(2)^{*}\left[w_{16}\right] \otimes B \otimes \Lambda\left(w_{6}, w_{8}\right)\left\{w_{7}\right\} \\
& E_{8}^{* \text { odd }} \cong P(3)^{*}\left[w_{16}\right] \otimes B \otimes\left\{w_{6} w_{7}, w_{8} w_{7}\right\} \\
& E_{16}^{* \text { odd }} \cong P(4)^{*}\left[w_{16}^{2}\right] \otimes B \otimes\left\{w_{8} w_{7}^{3}+w_{7} w_{6} w_{8}^{2}=D_{3} D_{4}, w_{8} w_{7} w_{16}=D_{4} w_{16}\right\}
\end{aligned}
$$

The next term is $E_{32}^{* \text { odd }} \cong P(5)^{*}\left[w_{16}^{2}\right] \otimes B\{a\}$, and finally $E_{64}^{*, o d d}=0$. Therefore all differentials have the form $d_{2^{r+1}}(x)=v_{r} \otimes Q_{r}(x)$ and the assumptions needed in the lemmas of Section 5 hold. The integral case can also be proved to satisfy these assumptions by similar but easier arguments. Indeed, $B P^{*}(B \operatorname{Spin}(9))$ is also computed in $[\mathrm{KY}]$. 
Theorem 7.2. In $B P^{*}(B D(4))$, the elements $\left[v_{1} \otimes w_{16}\right]$ and $\left[v_{2} \otimes w_{16}\right]$ are not transfers of $B P^{*}$-linear combinations of products of Chern classes.

\section{A 4-Dimensional PERmanent CyCle}

In this section, we show that the class $2 w_{4}$ in $H^{*}(B \operatorname{Spin}(n))_{(2)}$ is represented by a Chern class. A similar statement holds for the exceptional group $F_{4}$ and $p=3$.

Suppose that $G$ is a simply connected simple Lie group having $p$-torsion in $H^{*}(G)$. Then it is known that $G$ is 2 -connected and there is an element $x_{3} \in$ $H^{3}(G ; \mathbb{Z} / p)$ with $Q_{1} x_{3} \neq 0$. Consider the classifying space $B G$ and its cohomology. Denote by $y_{4}$ the transgression of $x_{3}$ in $H^{*}(B G ; \mathbb{Z} / p)$, so that $Q_{1}\left(y_{4}\right) \neq 0$. We shall denote the integral lift of $y_{4}$ to $H^{4}(B G)_{(p)}$ also by $y_{4}$. Then $y_{4}$ is not in the image from $B P^{*}(B G)$, and the following lemma is immediate.

Lemma 8.1. If $p y_{4} \in H^{4}(B G)_{(p)}$ is represented by a Chern class, then the kernel of the map $\bar{\rho}: C H^{2}(B G) / p \rightarrow H^{4}(B G ; \mathbb{Z} / p)$ is not injective.

First, we consider the case $G=\operatorname{Spin}(2 n+1)$ and $p=2$. The complex representation ring is

$$
R(\operatorname{Spin}(2 n+1)) \cong \mathbb{Z}\left[\lambda_{1}, \ldots, \lambda_{n-1}, \Delta_{C}\right],
$$

where $\lambda_{i}$ is the $i$-th elementary symmetric function in variables $z_{1}^{2}+z_{1}^{-2}, \ldots, z_{n}^{2}+$ $z_{n}^{-2}$ in $R(T) \cong \mathbb{Z}\left[z_{1}, z_{1}^{-1}, \ldots, z_{n}, z_{n}^{-1}\right]$ for the maximal torus $T$ in $\operatorname{Spin}(2 n+1)$. Consider the restriction to $R\left(S^{1}\right) \cong \mathbb{Z}\left[z_{1}, z_{1}^{-1}\right]$. Since

$$
\operatorname{Res}_{S^{1}}\left(\lambda_{1}\right)=z_{1}^{2}+z_{1}^{-2}+2(n-1),
$$

the total Chern class of this representation is

$$
\operatorname{Res}_{B S^{1}}\left(c\left(\lambda_{1}\right)\right)=(1+2 u)(1-2 u) .
$$

Therefore $4 u^{2} \in H^{*}\left(B S^{1}\right)$ is the restriction of a Chern class in $H^{*}(B \operatorname{Spin}(n+1))_{(2)}$.

On the other hand, consider the diagram:

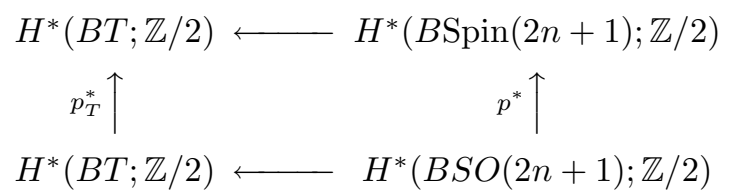

Here $p_{T}^{*}\left(u_{i}\right)=2 u_{i}$, and we see that $\operatorname{Res}_{B T}\left(w_{4}\right)=0$ in $H^{*}(B T ; \mathbb{Z} / 2)$. Thus

$$
\operatorname{Res}_{B S^{1}}\left(H^{4}\left(B \operatorname{Spin}(2 n+1)_{(2)}\right) \subset \mathbb{Z}_{(2)}\left\{2 u^{2}\right\} .\right.
$$

Therefore we see that for $G=\operatorname{Spin}(2 n+1)$ and $p=2$ the assumptions of Lemma 8.1 are satisfied. By naturality, all the groups $\operatorname{Spin}(n)$ for $n \geq 7$ satisfy the assumptions.

Next consider the case $G=F_{4}$ and $p=3$. The exceptional Lie group $F_{4}$ contains $\operatorname{Spin}(8)$ as a subgroup, and

$$
R\left(F_{4}\right) \cong R(\operatorname{Spin}(8))^{\Sigma_{3}}
$$

in $R(T) \cong \mathbb{Z}\left[z_{1}, z_{1}^{-1}, \ldots, z_{4}, z_{4}^{-1}\right]$ (for the details of the action of $\Sigma_{3}$, see [A] , Chapter 14). There is a 26 -dimensional irreducible representation $U$ of $F_{4}$, whose restriction to $\operatorname{Spin}(8)$ is $2+\lambda_{1}+\Delta^{+}+\Delta^{-}$, where $\Delta^{ \pm}$are the half spin representations of 
dimension 8. The weight of $\Delta^{ \pm}$is $\frac{1}{2}\left( \pm x_{1} \pm x_{2} \pm x_{3} \pm x_{4}\right)$, with an even number of minus signs for $\Delta^{+}$and an odd number for $\Delta^{-}$. Thus

$$
\operatorname{Res}_{S^{1}}\left(\Delta^{+}\right)=\sum_{\epsilon_{1} \epsilon_{2} \epsilon_{3} \epsilon_{4}=1} z_{1}^{\epsilon_{1}} z_{2}^{\epsilon_{2}} z_{3}^{\epsilon_{3}} z_{4}^{\epsilon_{4}}
$$

and similarly for $\Delta^{-}$. Restricting further to $S^{1}$, we obtain

$$
\operatorname{Res}_{S^{1}}(U)=z_{1}^{2}+z_{2}^{2}+8+8 z_{1}+8 z_{1}^{-1} .
$$

Therefore its total Chern class is

$$
\begin{aligned}
\operatorname{Res}_{B S^{1}}(c(U)) & =(1+2 u)(1-2 u)(1+u)^{8}(1-u)^{8} \\
& =\left(1-4 u^{2}\right)\left(1-u^{2}\right)^{8}=1-12 u^{2}+\cdots .
\end{aligned}
$$

Hence $3 y_{4} \in H^{4}\left(B F_{4}\right)_{(3)} \cong \mathbb{Z}_{(3)}$ is represented by a Chern class. Thus we get the following therorem.

Theorem 8.2. Let $G=\operatorname{Spin}(n), n \geq 7$ and $p=2$, or $G=F_{4}$ and $p=3$. The kernels of the maps

$$
\begin{aligned}
& C H^{2}(B G) /(p) \rightarrow H^{4}(B G ; \mathbb{Z} / p), \\
& C H^{3}(B G \times B \mathbb{Z} / p)_{(p)} \rightarrow H^{6}(B G \times B \mathbb{Z} / p)_{(p)}
\end{aligned}
$$

are both non-zero.

\section{REFERENCES}

[A] J. F. Adams. Lectures on exceptional Lie groups, edited by Z. Mahmud and M. Mimura. Chicago Lectures in Mathematics, The University of Chicago Press, 1996. MR 98b:22001

[BC] D. J. Benson and J. F. Carlson. The cohomology of extraspecial groups. Bull. London Math. Soc. 24 (1992), 209-235; erratum 25 (1993), 498. MR 93b:20087. MR 94f:20099

[GL] D. J. Green and I. J. Leary. The spectrum of the Chern subring. Comm. Math. Helv. 73 (1998), 406-426. MR 99j:20070

[GM] D. Green and P. A. Minh. Transfer and Chern classes for extraspecial p-groups. In: A. Adem, J. F. Carlson, S. B. Priddy and P. J. Webb, Eds. Group representations: cohomology, group actions and topology (Seattle, WA, 1996). Proc. Sympos. Pure Math. 63, pp. 245-255, Amer. Math. Soc., Providence, RI, 1998. MR 99d:20083

[HK] M. Harada and A. Kono. On the integral cohomology of extraspecial 2-groups. Proceedings of the Northwestern conference on cohomology of groups (Evanston, Ill., 1985). J. Pure Appl. Algebra 44 (1987), 215-219. MR 88b:20084

[HKR] M. J. Hopkins, N. J. Kuhn, and D. C. Ravenel. Morava $K$-theories of classifying spaces and generalized characters for finite groups. In: J. Aguadé, M. Castellet and F. R. Cohen, Eds. Algebraic topology (Sant Feliu de Guíxols, 1990). Lecture Notes in Math. 1509 (1992), Springer, 186-209. MR 93k:55008

[KY] A. Kono and N. Yagita. Brown-Peterson and ordinary cohomology theories of classifying spaces for compact Lie groups. Trans. Amer. Math. Soc. 339 (1993), 781-798. MR 93m:55006

[K] I. Kriz. Morava $K$-theory of classifying spaces: some calculations. Topology 36 (1997), 1247-1273. MR 99a:55016

[Q] D. G. Quillen. The mod 2 cohomology rings of extra-special 2-groups and the spinor groups. Math. Ann. 194 (1971), 197-212. MR 44:7582

[RWY] D. C. Ravenel, W. S. Wilson and N. Yagita. Brown-Peterson cohomology from Morava $K$-theory. $K$-Theory 15 (1998), 147-199. CMP 99:02

[S] B. Schuster. On the Morava K-theory of some finite 2-groups. Math. Proc. Cambridge Philos. Soc. 121 (1997), 7-13. MR 97i:55008

[T1] B. Totaro. Torsion algebraic cycles and complex cobordism. J. Amer. Math. Soc. 10 (1997), 467-493. MR 98a:14012

[T2] B. Totaro. The Chow ring of classifying spaces. To appear. 
[Y1] N. Yagita. Cohomology for groups of $\operatorname{rank}_{p}(G)=2$ and Brown-Peterson cohomology. $J$. Math. Soc. Japan 45 (1993), 627-644. MR 94k:55008

[Y2] N. Yagita. On relations between Brown-Peterson cohomology and the ordinary mod $p$ cohomology theory. Kodai Math.J 7 (1984), 273-285. MR 85g:55007

FB 7 Mathematik, Bergische Universität-Gesamthochschule Wuppertal, 42097 WupPERTAL, GERMANY

E-mail address: schuster@math.uni-wuppertal.de

Department of Mathematics, Faculty of Education, Ibaraki University, Mito, IbaraKI, JAPAN

E-mail address: yagita@mito.ipc.ibaraki.ac.jp 